\title{
A Precise Measurement of Temperature Difference Using Thermopiles
}

\author{
B. J. Huang \\ Department of Mechanical Engineering, \\ National Taiwan University, \\ Taipei, Taiwan
}

\begin{abstract}
- The theory of thermopiles from multipairs of thermocouples for precise measurement of temperature difference $\Delta T$ was derived, and a calibration and signal conversion method using the theory was developed in the present study. The standard $e m f-T$ relation, $E(T)$ or $T(E)$, for thermopile was defined based upon the thermopile theory and can be determined by calibration against a precise thermometer and potentiometer. Then, a midpoint extrapolation method was applied to the emf-T curve of the thermopile to convert the measured emf signal to $\Delta T$ accurately. For purposes of illustration, a thermopile made from five pairs of T-type thermocouples was designed and calibrated by using the present method. It is shown that the uncertainty of this five-pair, T-type thermopile can be as low as $\pm 0.073^{\circ} \mathrm{C}$, which has uncertainty less than $\pm 2 \%$ for a $\Delta T$ of about $\pm 3.5^{\circ} \mathrm{C}$.
\end{abstract}

Keywords: temperature measurement technique, thermopiles, thermocouples

\section{INTRODUCTION}

For many heat transfer experiments in the energy or process industry, one may have to measure a small temperature difference $\Delta T$ to within certain degree of accuracy. This may cause some difficulties, since accurate measurement of small $\Delta T$ requires precise instruments or use of special techniques. For instance, in the efficiency test of a solar collector, the uncertainty of $\leq \pm 2 \%$ in the temperature difference measurement across the collector is sometimes required in order to improve the overall accuracy in the efficiency measurement [1]. This means that the allowable uncertainty is about $\pm 0.1^{\circ} \mathrm{C}$ for $\Delta T=5^{\circ} \mathrm{C}$. Although we can determine $\Delta T$ by differencing two separately measured temperatures made at the inlet and outlet of the collector using thermocouple thermometers, this accuracy requirement can hardly be met, since the uncertainty of conventional thermocouple thermometer can be as large as $\pm 0.7^{\circ} \mathrm{C}$ or even larger.

Precise but expensive temperature-measuring techniques, such as a high-precision platinum resistance thermometer or a quartz thermometer, can be used to solve the above problem. However, in addition to the cost consideration, their applications are sometimes restricted by the difficulty in installing the special probes to the desired positions. Therefore, thermopile made from multipairs of thermocouples connected in series developed by Schnurr [2] appears to be a promising solution.

In the calibration of thermopile, Schnurr [2] used a precise platinum resistance thermometer to measure the temperatures of two baths inside which two probes of a thermopile were separately inserted. During calibration, one of the baths was set at the designated base temperature, and the second bath was adjusted to obtain the desired temperature difference $\Delta T$. The $e m f$ of the thermopile was then recorded at each checkpoint to obtain a calibration chart or table. During application, $\Delta T$ can be converted directly from the calibration chart or table by use of the output emf signal of the thermopile that is measured by a precise potentiometer. Apparently, this kind of calibration and signal conversion method for a thermopile is tedious, and the accuracy may be reduced because of the facts that the $e m f-\Delta T$ calibration chart or table for various base temperatures is needed, and, thus, interpolation in the charts may be required sometimes. This may explain why the major application areas of thermopiles are still in the radiation-sensitive elements [3] and the heat flux sensors [4].

Instead of using the $e m f-\Delta T$ calibration chart directly, the new method developed in the present study basically uses the standard emf-T relationship of the thermopile, that is, $E(T)$ or $T(E)$, which was theoretically derived from the thermopile theory and can be calibrated first by use of a precise thermometer. Then, a midpoint extrapolation can be applied to the $e m f-T$ curve of the thermopile to convert the measured $e m f$ signal into the temperature difference $\Delta T$ accurately. The uncertainty for a particular thermopile used in this way was estimated and is reported in this article.

\section{THERMOPILE THEORY}

Thermocouple thermometer is a widely used temperature measuring device. The measurement error of a conventional thermocouple thermometer mainly comes from two sources: (1) thermocouple wire error that is caused by the variation of wire composition during manufacturing process or deterioration of material properties of wire or junction because of thermal cycling during applications so that the actual $e m f-T$ relation deviates from the standard one already attached either in hardware or software inside the thermometer; and (2) the potentiometer error in measuring the output emf. The former one can be as large as $\pm 0.5^{\circ} \mathrm{C}$ for T-type thermocouples, for example. The latter one can be as large as $\pm 5 \mu \mathrm{V}$ for a $5 \frac{1}{2}$ digit potentiometer.

According to the above analysis, an efficient method for measuring small temperature difference using thermocouple

Address correspondence to Professor B. J. Huang, Department of Mechanical Engineering, National Taiwan University, Taipei, Taiwan 10764.

Experimental Thermal and Fluid Science 1990; 3:265-271

(C) 1990 by Elsevier Science Publishing Co., Inc., 655 Avenue of the Americas, New York, NY 10010

$0894-1777 / 90 / \$ 3.50$ 


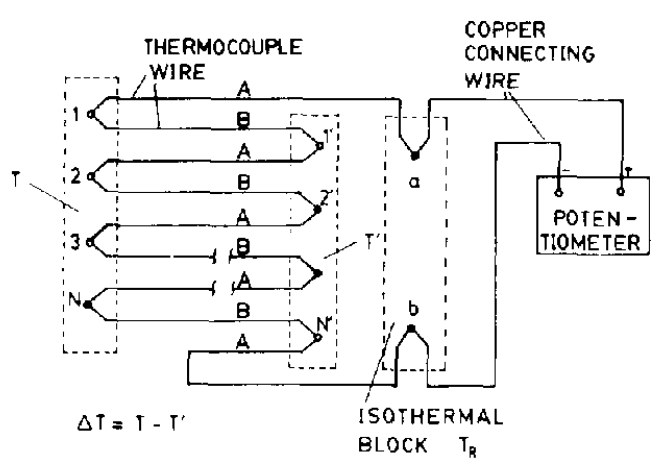

Figure 1. Thermopile circuit.

is: (1) to install two thermocouple junctions at the desired two points to directly read the corresponding output voltage difference; (2) to connect $N$ pairs of thermocouple wires in series to form a thermopile in order to enhance the thermocouple output emf to reduce the potentiometer error by $N$ times; (3) to calibrate the thermopile using a precise thermometer (see Fig. 1). The development of a new method of calibration and signal conversion based upon a thermopile theory is thus necessary.

Although thermopiles is a rather old technique, the theory of thermopiles for $\Delta T$ measurements is, however, still not available in literature. To fulfill this gap, the thermopile theory is first presented below.

By thermoelectric theory, the total thermopile output emf for $N$ pairs of thermocouple wires connected in series is, by referring to Fig. 1,

$$
\begin{aligned}
\epsilon_{a}-\epsilon_{1} & =-\int_{T_{a}}^{T_{1}} \alpha_{A, 1} d T \\
& =-\int_{T_{a}}^{T_{1}^{\prime}} \alpha_{A, 1} d T+\int_{T_{1}}^{T_{1}^{\prime}} \alpha_{A, 1} d T \\
\epsilon_{1}-\epsilon_{1}^{\prime} & =-\int_{T_{1}}^{T_{1}^{\prime}} \alpha_{B, 1} d T \\
\epsilon_{1}^{\prime}-\epsilon_{2} & =-\int_{T_{1}^{\prime}}^{T_{2}} \alpha_{A, 2} d T=\int_{T_{2}}^{T_{1}^{\prime}} \alpha_{A, 2} d T \\
\epsilon_{2}-\epsilon_{2}^{\prime} & =-\int_{T_{2}}^{T_{2}^{\prime}} \alpha_{B, 2} d T \\
\ldots & =\cdots \\
\cdots & =\cdots \\
\epsilon_{N-1}^{\prime}-\epsilon_{N} & =-\int_{T_{N-1}^{\prime}}^{T_{N}} \alpha_{A, N} d T=\int_{T_{N}}^{T_{N-1}^{\prime}} \alpha_{A, N} d T \\
\epsilon_{N}^{\prime}-\epsilon_{b}= & -\int_{T_{N}^{\prime}}^{T_{N}^{\prime}} \alpha_{A, N} d T=\int_{T_{b}}^{T_{N}^{\prime}} \alpha_{A, N} d T \\
\epsilon_{N}^{\prime} & =-\alpha_{T_{N}} d T
\end{aligned}
$$

where $\alpha_{A, i}$ and $\alpha_{B, i}$ are the absolute thermoelectric power of wires $A$ and $B$ of the thermocouple, respectively, for the $i$ th pair of the thermopile. Since the measuring junctions on each sides are at the same temperatures, we have $T_{1}=T_{2}=$ $\cdots=T_{N}=T$ and $T_{1}^{\prime}=T_{2}^{\prime}=\cdots=T_{N}^{\prime}=T^{\prime}$. Therefore, if we assume further that $T_{a}=T_{b}=T_{R}$, the total thermopile output emf, $E_{T}$, becomes

$$
\begin{aligned}
E_{T}= & \epsilon_{a}-\epsilon_{b}=\int_{T_{R}}^{T^{\prime}}\left(\alpha_{A, N}-\alpha_{A, 1}\right) d T \\
& +\int_{T}^{T^{\prime}}\left[\left(\alpha_{A, 1}-\alpha_{B, 1}\right)+\left(\alpha_{A, 2}-\alpha_{B, 2}\right)+\cdots\right. \\
& \left.+\left(\alpha_{A, N}-\alpha_{B, N}\right)\right] d T=\int_{T_{R}}^{T^{\prime}}\left(\alpha_{A, N}-\alpha_{A, 1}\right) d T \\
& -\int_{T_{R}}^{T}\left(\alpha_{A B, 1}+\alpha_{A B, 2}+\cdots+\alpha_{A B, N}\right) d T \\
& +\int_{T_{R}}^{T^{\prime}}\left(\alpha_{A B, 1}+\alpha_{A B, 2}+\cdots+\alpha_{A B, N}\right) d T
\end{aligned}
$$

where $\alpha_{A B, i}$ represents the Seebeck effect induced by the $i$ th thermocouple pair of the thermopile that is defined as

$$
\alpha_{A B, i} \equiv \alpha_{A, i}-\alpha_{B, i}
$$

It has to be noted that the above assumption of $T_{a}=T_{b}=T_{R}$ always holds in practice, if the two connecting terminals of the thermopile, $a$ and $b$, are all mounted on a well-designed isothermal block with uniform temperature distribution.

In practice, the first integral of the right-hand side of Eq. (2) is negligible as compared to the sum of the other two integrals. Moreover, if the length and size and material compositions of the first and the last wires of $\mathrm{A}$ are approximately the same, we have $\alpha_{A, 1}=\alpha_{A, N}$, and Eq. (2) turns out to be

$$
\begin{aligned}
E_{T}= & \epsilon_{a}-\epsilon_{b} \\
= & -\int_{T_{R}}^{T}\left(\alpha_{A B, 1}+\alpha_{A B, 2}+\cdots+\alpha_{A B, N}\right) d T \\
& +\int_{T_{R}}^{T^{\prime}}\left(\alpha_{A B, 1}+\alpha_{A B, 2}+\cdots+\alpha_{A B, N}\right) d T \\
= & N\left[-\int_{T_{R}}^{T} \bar{\alpha}_{A B} d T+\int_{T_{R}}^{T^{\prime}} \bar{\alpha}_{A B} d T\right] .
\end{aligned}
$$

where $\bar{\alpha}_{A B}$ is the average Seebeck effect for the thermopile that is defined as

$$
\bar{\alpha}_{A B}(T)=\frac{\alpha_{A B, 1}+\alpha_{A B, 2}+\cdots+\alpha_{A B, N}}{N} .
$$

If we further set the reference temperature $T_{R}$ to be the ice point, that is, $T_{R}=0^{\circ} \mathrm{C}$, then a standard emf-T relation for the thermopile can be defined in accordance with Eq. (4):

$$
E(T) \equiv-\int_{0^{\circ} \mathrm{C}}^{T} \bar{\alpha}_{A B} d T
$$

Therefore, Eq. (4) becomes

$$
E_{T}=\epsilon_{a}-\epsilon_{b}=N\left[E(T)-E\left(T^{\prime}\right)\right]
$$


or, written in terms of the specific output emf of thermopile, $\Delta E$,

$$
\Delta E=\frac{E_{T}}{N}=E(T)-E\left(T^{\prime}\right)
$$

Equation (8) essentially forms the basic theory of thermopiles in which the standard $e m f-T$ relation $E(T)$ for thermopiles is defined in Eq. (6).

It can be seen from Eq. (7) or (8) that the thermopile output emf, $E_{T}$ or $\Delta E$, is independent of the isothermal block temperature $T_{R}$. Therefore, it does not require any reference temperature compensation when using thermopile for $\Delta T$ measurement.

Apparently, the standard $e m f-T$ relation, $E(T)$, defined in Eq. (6) can be experimentally determined by a calibration, and the data can be collected in a emf-T table similar to the NBS standard tables for single thermocouple wires [5]. For ease of application, an empirical equation for $E(T)$ may also be derived.

In the implementation of thermopile, we can use the calibrated $E(T)$ relation to convert the measured emf signal of the thermopile, $\Delta E$, into the temperature difference $\Delta T$ through the implicit relation shown in $\mathrm{Eq}$. (8). However, unless the calibrated $E(T)$ relation is linear, it is impossible to write $\mathrm{Eq}$. (8) in the form $\Delta E=f(\Delta T)$. Therefore, the conversion from $\Delta E$ to $\Delta T$ is difficult and needs a special signal conversion method. To assure good accuracy, a signal conversion method will be developed in the present study. Before doing this, we will first show how to calibrate a thermopile.

\section{THERMOPILE CALIBRATION}

To illustrate how the thermopile can be calibrated and implemented using the theory derived above, a thermopile consisting of five pairs of T-type thermocouple and connected in series was designed for the measurement of small $\Delta T$. Since the first and the fifth $\mathrm{Cu}$ wires connecting to the isothermal block were made with the same size and length from the same $\mathrm{Cu}$ spool of the T-type thermocouple, their thermoelectric properties will be approximately identical, that is, $\alpha_{A, 1} \approx \alpha_{A, 5}$. Thus, Eq. (4) holds for the thermopile.

It should be emphasized here that the methodology developed in the present study is applicable to any thermopile made from multipairs of any type of thermocouple wires. The use of five pairs of $\mathrm{T}$-type thermocouples for the thermopile is just for illustrative purposes.

The first step to implement the thermopile is to calibrate the thermopile to determine the emf-T relation, $E(T)$, according to the theory described above. The calibration procedure is the same as that of obtaining the NBS standard table for single thermocouple wires [5].

To do so, one probe of the thermopile has to be immersed in a ice bath. In the present study, an Omega ice point device (with temperature variation $< \pm 0.1^{\circ} \mathrm{C}$ ) was used. That is, the calibration will be performed at the condition of $T^{\prime}=T_{R}=0^{\circ} \mathrm{C}$. Thus, $E\left(T^{\prime}\right)=E(0)=0$, and $E(T)$ can be determined using $\mathrm{Eq}$. (8), that is, $E(T)=E_{T} / N$ (see Fig. 2 ). The other probe of the thermopile has to be immersed in another bath, and the temperature measurement of the bath using precise thermometer is required. In the present study, a well-insulated constant-temperature bath was used, and an HP 2804 precise quartz thermometer was used to measure the bath temperature. The temperature variation of the bath was less

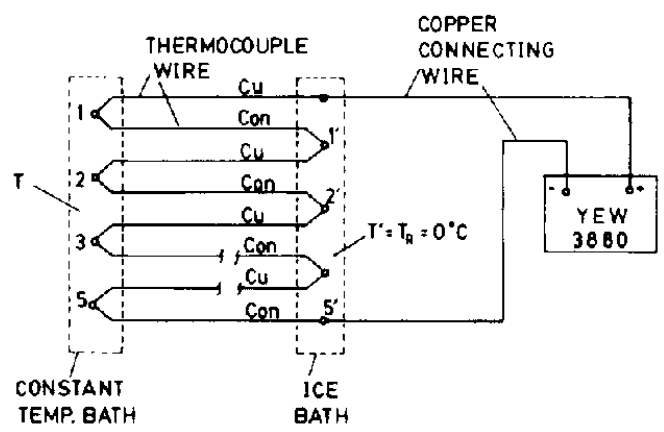

Figure 2. Calibration of a thermopile made from five pairs of T-type thermocouples.

than $\pm 0.01^{\circ} \mathrm{C}$, and, thus, its resultant error is negligible. An HP 2804 precise quartz thermometer provides accurate temperature measurement with uncertainty of $\pm 0.04^{\circ} \mathrm{C}$ and, thus, was used as the standard device for the calibration. To read the total thermopile emf, $E_{T}$, the $5 \frac{1}{2}$ digit potentiometer in the Yokogawa YEW-3880 recorder with uncertainty of $\pm 5 \mu \mathrm{V}$ was used. The thermopile output emf at $T_{R}=0^{\circ} \mathrm{C}$, that is, $E(T)$ as defined by Eq. (6), can then be obtained by dividing $E_{T}$ by $\mathrm{N}$. That is, $E(T)=E_{T} / 5$ from Eq. (8), since $E\left(T^{\prime}\right)=E\left(0^{\circ} \mathrm{C}\right)=0$ for the present arrangement.

The calibration was performed at temperatures ranging from 10 to $90^{\circ} \mathrm{C}$. The final results are shown in Fig. 3, and a third-order function was fitted to the data.

$$
\begin{array}{r}
T(E)=-.085369+25.991877 E-.768954 E^{2}+.037124 E^{3} \\
\text { for } 10 \leq T \leq 90^{\circ} \mathrm{C} \quad(9)
\end{array}
$$

where $E$ is in $m v, T$ is in ${ }^{\circ} \mathrm{C}$. It can be seen from Fig. 4 that the fitted $e m f-T$ relation for the thermopile has an error of $\leq \pm 0.05^{\circ} \mathrm{C}$. This calibration relationship will be used for the determination of temperature difference. Here, we use the functional relation $T(E)$ instead of $E(T)$, defined in Eq. (6) for convenience of the signal conversion during implementation.

\section{METHOD OF SIGNAL CONVERSION}

To apply the thermopile to the measurement of $\Delta T$, the two probes of the thermopile will be separately inserted to two desired positions, and the total emf signal of the thermopile will

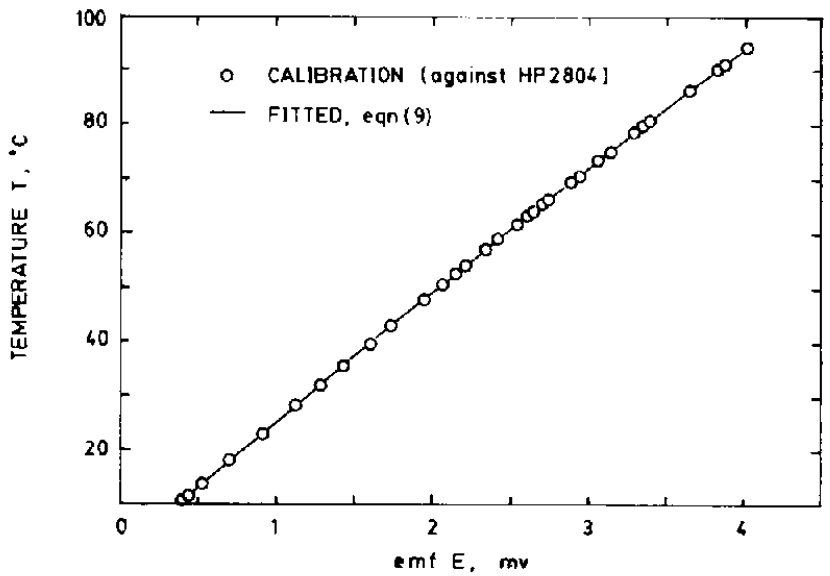

Figure 3. Calibration curve of thermopile. 


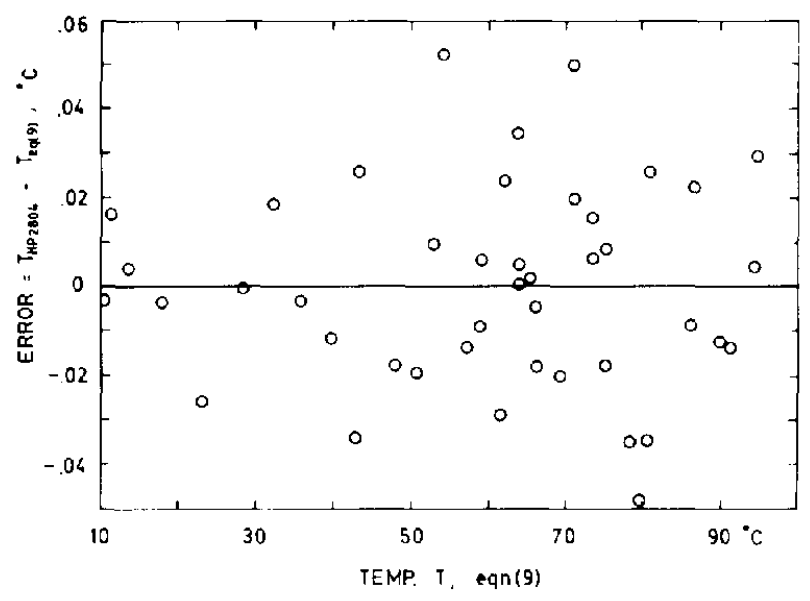

Figure 4. Calibration error of thermopile.

be recorded by a potentiometer. In addition, the two connecting terminals of the thermopile, $a$ and $b$, should be mounted on an isothermal block such that $T_{a}=T_{b}=T_{R}$, which can be any value (not necessary to be ice point, $0^{\circ} \mathrm{C}$ ) (see Fig. 1). Equation (8) can then be used to determine $\Delta T$. For convenience, Eq. (8) can be written in an alternate form

$$
\Delta T \equiv T-T^{\prime}=T(E)-T\left(E^{\prime}\right) .
$$

Since only the total output emf of the thermopile, $E_{T}$, or the emf difference $\Delta E\left(=E_{T} / N\right)$ corresponding to $\Delta T$ can be measured, Eq. (10) can be directly used for the conversion of temperature difference $\Delta T$ only if the emf-temperature relation $T(E)$ is linear. That is, if $T(E)$ is linear, that is, $T(E)=a+b E$, where $a$ and $b$ are constants, then Eq. (10) becomes

$$
\Delta T=b E-b E^{\prime}=b \Delta E=T(\Delta E) .
$$

In this case, we need only to substitute $\Delta E$ directly into the emf $f T$ relation of the thermopile to obtain $\Delta T$. This method of signal conversion was frequently used by many experimenters. Unfortunately, the emf-T relation, $T(E)$, for thermopile is usually not linear. Figure 5 shows that significant error will result if a linear approximation, instead of Eq. (9),

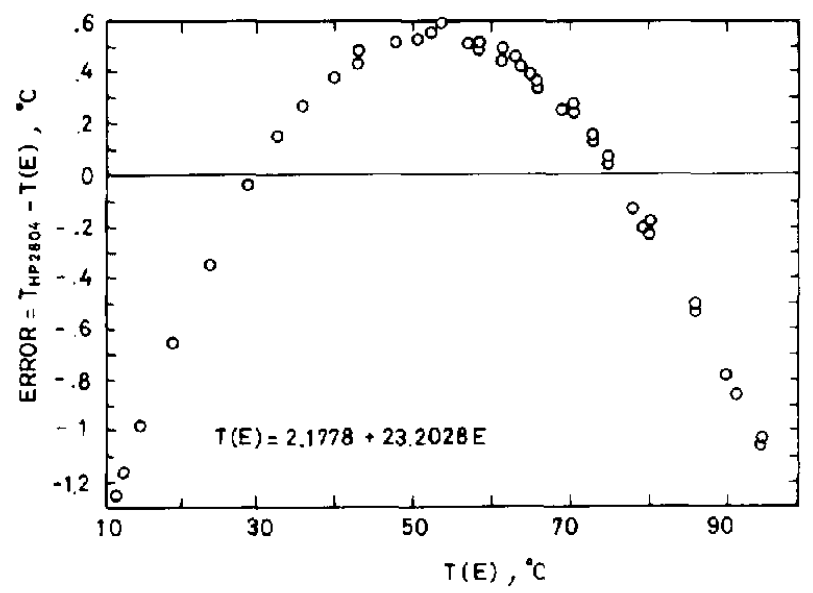

Figure 5. Calibration error caused by linear approximation.

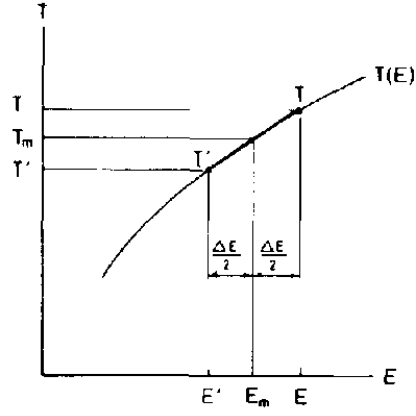

Figure 6. Extrapolation from midpoint.

is used. The linear approximation for the present thermopile is $T(E)=2.1778+23.2028 E$, which was obtained by fitting the calibration data to a linear function. To overcome this problem, method of extrapolation starting from midpoint was developed in the present study to determine the corresponding $e m f$ at $T$ and $T^{\prime}$, that is, $E$ and $E^{\prime}$.

If one can estimate the mean temperature $T_{m}$ of the two points using other conventional instrument, then the corresponding emf at this mean temperature, $E_{m}$, can be computed by using Eq. (9) with iteration scheme. Starting from this midpoint, we can extrapolate to both directions in the $T(E)$ curve using the measured thermopile output $e m f, \Delta E$. For simplification, half-size steps, that is, $\Delta E / 2$, were used in the present study. Therefore, the two points, $(E, T)$ and $\left(E^{\prime}, T^{\prime}\right)$ can be located on the $T(E)$ curve of the thermopile by the following relations (refer to Fig. 6):

$$
E=E_{m}+\frac{\Delta E}{2} \quad E^{\prime}=E_{m} \frac{\Delta E}{2} .
$$

Substituting $E$ and $E^{\prime}$ into Eq. (10), we can then determine $\Delta T$.

The final problem left is the determination of $T_{m}$. A simple solution is that we can install an additional two thermocouples at the two desired points and use a conventional thermocouple thermometer to measure $T$ and $T^{\prime}$ separately and then take the average. That is, $T_{m}=\left(T+T^{\prime}\right) / 2$. Although $\Delta T$ can also be determined by this direct measurement of $T$ and $T^{\prime}$, the accuracy is low, as described previously, and, thus, refinement is required. In other words, these two temperatures are used only for locating the mean point $\left(E_{m}, T_{m}\right)$. Of course, the error in measuring $T_{m}$ is relatively large. But its effect on the resultant uncertainty of temperature difference measurement can be estimated and shown to be very small. This will be discussed later.

We can summarize the signal conversion procedure for the thermopile as follows:

1. Measure $T$ and $T^{\prime}$ using a conventional thermometer.

2. Determine $T_{m}=\left(T+T^{\prime}\right) / 2$.

3. Determine $\Delta E\left(=E_{T} / N\right)$ by measuring $E_{T}$ using a potentiometer.

4. Convert $T_{m}$ to $E_{m}$ by Eq. (9) using iteration.

5. Determine $E$ and $E^{\prime}$ using Eq. (12).

6. Convert $E$ and $E^{\prime}$ into $T$ and $T^{\prime}$ by Eq. (9) using iteration.

7. Compute $\Delta T\left(=T-T^{\prime}\right)$.

Following the above procedures, the conversion chart for the thermopile can be determined. Figure 7 shows some of the 
results. If a computer-controlled data acquisition system was used in the measurement, this signal conversion can easily be carried out by software.

Since for small $\Delta T$, the line segment between the two points $(E, T)$ and $\left(E^{\prime}, T^{\prime}\right)$ on the emf-T curve of the thermopile is approximately linear, the errors resulting from the extrapolation can be negligible (see Fig. 6). The main source of error comes from the error in measuring $T_{m}$. However, it can be shown that for the error of $T_{m}$ at $\pm 2^{\circ} \mathrm{C}$, an overall error of $\Delta T \leq \pm 0.5 \%$ will result. See Fig. 8, where the error $e(\Delta T)$ was defined as

$$
e(\Delta T)=\Delta T^{*}-\Delta T
$$

or, in relative form,

$$
\frac{e(\Delta T)}{\Delta T}=\frac{\Delta T^{*}-\Delta T}{\Delta T}
$$

where $\Delta T^{*}$ is the temperature difference for the mean temperature $T_{m}^{*}$, which varies within $T_{m} \pm 2^{\circ} \mathrm{C} ; \Delta T$ is the converted temperature difference using the estimated value $T_{m}=\left(T+T^{\prime}\right) / 2$. Here, the variation of $\pm 2{ }^{\circ} \mathrm{C}$ for $T_{m}$ is used for the uncertainty estimation, since the uncertainty in measuring $T$ and $T^{\prime}$ can be as large as $\pm 1^{\circ} \mathrm{C}$ if a conventional thermocouple thermometer was used.

\section{THERMOPILE UNCERTAINTY}

It can be seen that the thermopile uncertainty mainly results from three sources: (1) the uncertainty of the $e m f-T$ relation, Eq. (9); (2) the uncertainty of potentiometer used to detect the thermopile output $e m f, E_{T}$; and (3) the uncertainty induced by the inaccurate estimation of $T_{m}$ when using a conventional
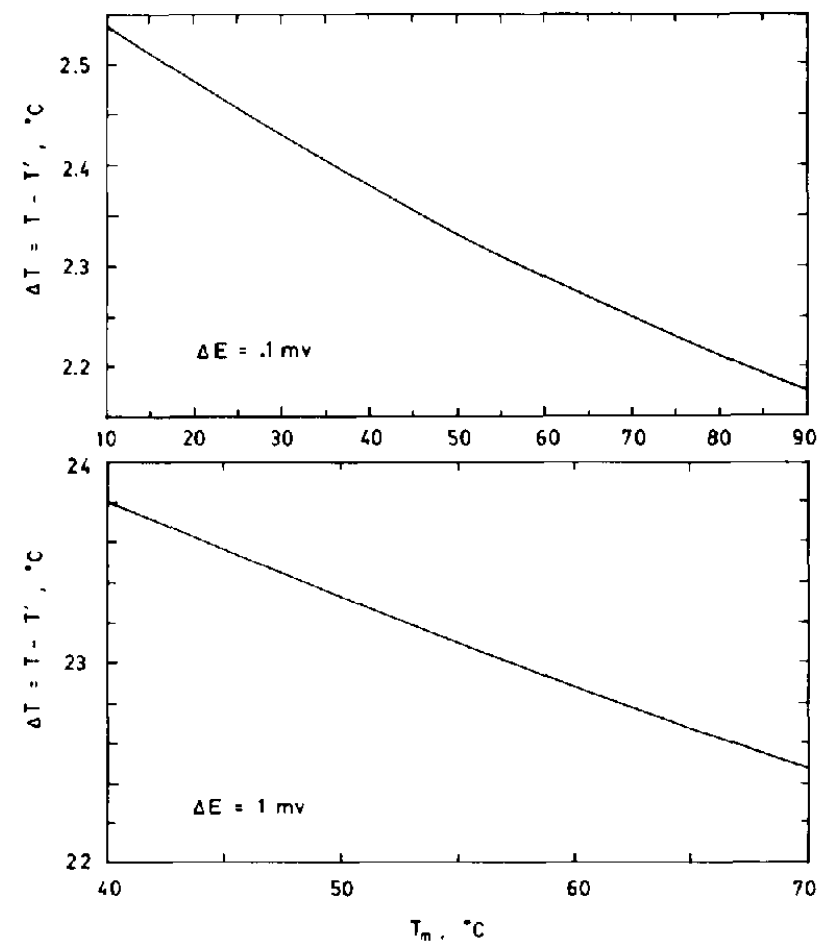

Figure 7. Conversion chart of thermopile.

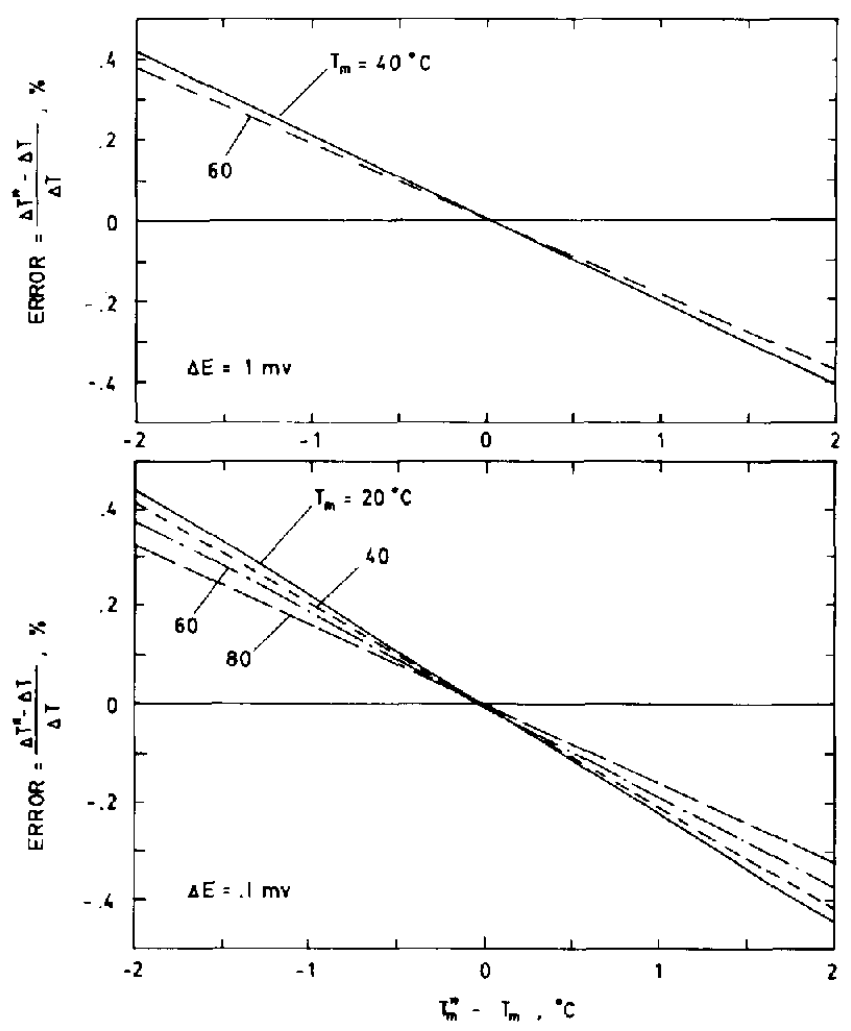

Figure 8. Error in measurement of $\Delta T$ caused by uncertainty of $T_{m}$.

thermometer. The overall uncertainty of the present thermopile can be evaluated. For illustrative purposes, the uncertainty evaluation is carried out for the five-pairs of T-type thermopile particularly made for the present study.

\section{Uncertainty Because of the $e m f-T$ Calibration Curve}

It can be seen from Fig. 4 that the fitted relation, Eq. (9), has an uncertainty of $\pm 0.05^{\circ} \mathrm{C}$. Since this calibration curve is performed against the standard device, a HP 2804 quartz thermometer, with uncertainty of $\pm 0.04^{\circ} \mathrm{C}$, the combined uncertainty because of the $e m f-T$ relation is

$$
\sqrt{0.05^{2}+0.04^{4}}=0.06^{\circ} \mathrm{C}
$$

\section{Uncertainty Because of the Potentiometer}

From the relation of Eq. (10), the combined uncertainty for the measurement of $\Delta T$ by using the $e m f-T$ calibration curve can be evaluated by the following equation $[6,7]$ :

$$
w_{\Delta T}^{2}=\left(\frac{d T}{d E}\right)_{E}^{2} w_{E}^{2}+\left(\frac{d T}{d E}\right)_{E^{\prime}}^{2} w_{E^{\prime}}^{2}
$$

where $w_{E}$ and $w_{E^{\prime}}$ are the uncertainties in locating $E$ and $E^{\prime}$, respectively. Since only the overall output emf $\Delta E\left(=E-E^{\prime}\right)$, instead of $E$ and $E^{\prime}$, can be measured for thermopile, it can be assumed in a rather conservative manner that these uncer- 
tainties are the same as the uncertainty in the measurement of $\Delta E$.

The uncertainty of the $5 \frac{1}{2}$ digit potentiometer of YEW3880 used for the detection of thermopile output emf is $\pm 5 \mu \mathrm{V}$. Since $\Delta E=E_{T} / 5$, we further obtain

$$
w_{E} \approx w_{E^{\prime}} \approx w_{\Delta E}=\frac{w_{E_{T}}}{5}=\frac{5 \mu V}{5}=1 \mu V=0.001 m v
$$

Note that the uncertainty in measuring the output emf of the thermopile can be reduced by $N$ times if $N$ pairs of thermocouples are used to make the thermopile. Here, $(d T / d E)_{E}$ and $(d T / d E)_{E}$ are the slopes of the emf-T curve at points $(E, T)$ and $\left(E^{\prime}, T^{\prime}\right)$, respectively. An estimation from Fig. 3 shows that $(d T / d E)_{E} \approx(d T / d E)_{E^{\prime}} \approx 26^{\circ} \mathrm{C} / m v$. Therefore, we obtain from Eq. (15)

$$
\begin{aligned}
w_{\Delta T} & =\sqrt{\left(26 \frac{{ }^{\circ} \mathrm{C}}{m v} \times 0.001 m v\right)^{2}+\left(26 \frac{{ }^{\circ} \mathrm{C}}{m v} \times 0.001 m v\right)^{2}} \\
& =\sqrt{0.026^{2}+0.026^{2}}=0.037^{\circ} \mathrm{C} .
\end{aligned}
$$

\section{Uncertainty Induced by Inaccurate Estimation of $T_{m}$}

It has been shown from Fig. 8 that the resulting uncertainty because of the estimation error of $T_{m}$ is $\pm 0.5 \%$ if the maximum uncertainty in estimating $T_{m}$ is $\pm 2^{\circ} \mathrm{C}$, which can be easily obtained by using conventional thermocouple thermometer. For a temperature difference of $10^{\circ} \mathrm{C}$, this error is $\pm 0.05^{\circ} \mathrm{C}$.

It should be noted that uncertainty of $T_{m}$ can be further reduced if a more precise thermometer is used to determine $T_{m}$. For instance, if the thermocouple thermometer used to measure $T$ and $T^{\prime}$ is also calibrated against an HP 2804 quartz thermometer to obtain a new $e m f-T$ relation, the uncertainty in estimating $T_{m}$ can be further reduced, and so does the resulting uncertainty of $\Delta T$.

Since the above three kinds of uncertainties are mutually independent, the most probable error of the thermopile for measuring a temperature difference $\Delta T$ is

$$
\sqrt{.06^{2}+.037^{2}+(.005 \times \Delta T)^{2}} .
$$

Hence, the overall uncertainty for the present thermopile in measuring various $\Delta T$ is shown as follows:

\begin{tabular}{rrr}
$\Delta T^{\circ} \mathrm{C}$ & Uncertainty, ${ }^{\circ} \mathrm{C}$ & Uncertainty, $\%$ \\
\hline \pm 1 & \pm .070 & \pm 7.0 \\
\pm 2 & \pm .071 & \pm 3.6 \\
\pm 3 & \pm .072 & \pm 2.4 \\
\pm 4 & \pm .073 & \pm 1.8 \\
\pm 5 & \pm .075 & \pm 1.5 \\
\pm 10 & \pm .086 & \pm 0.9
\end{tabular}

It can be seen from these results that very small $\Delta T$ can be detected by the thermopile with high accuracy. The uncertainty of the present thermopile in detecting $\Delta T$ can be as low as $\pm 0.073^{\circ} \mathrm{C}$ which is at $\pm 2 \%$ accuracy level for temperature difference of about $\pm 3.5^{\circ} \mathrm{C}$.
The practical significance of the thermopiles used in the measurement of small temperature difference based upon the present development mainly lies in the methods of calibration (including the derivation of a new thermopile theory) and signal conversion, from which high accuracy can be ensured. Besides, the ease of installation and low cost makes this old technique quite useful indeed.

\section{CONCLUSION}

The theory of thermopiles for precision measurement of temperature difference $\Delta T$ was derived, and a calibration and signal conversion method based on this theory was developed in the present study. The standard emf-T relation, $E(T)$ or $T(E)$, for the thermopile was defined based upon the thermopile theory and was determined by calibration using a precise thermometer. Then, a midpoint extrapolation was applied to the $e m f-T$ curve to convert the measured emf signal into $\Delta T$ accurately. It is shown that the uncertainty of a thermopile, which was made from five pairs of T-type thermocouples, can be as low as $\pm 0.073^{\circ} \mathrm{C}$, which is at $\pm 2 \%$ accuracy level for a $\Delta T$ of about $\pm 3.5^{\circ} \mathrm{C}$.

It should also be emphasized here that the technique developed in the present study is applicable to any thermopile made from multipairs of any type of thermocouple wires. The use of five pairs of T-type thermocouple as the thermopile was just for illustrative purposes. Nevertheless, high accuracy can always be obtained for thermopiles made from different kind of thermocouples.

The overall accuracy of the thermopile can be further improved by (1) using more pairs of thermocouples in a thermopile, (2) using precise thermometer with much higher accuracy in the calibration of $e m f-T$ relation of thermopile, $E(T)$, and (3) using potentiometer with higher accuracy to measure the output emf of the thermopile. In addition, it can also be noted that $\Delta T$ measurement using thermopile associated with the present calibration and signal conversion method does not require any reference temperature compensation and thus costs are reduced.

The present study was supported by the Energy Committee, Ministry of Economic Affairs, Taiwan, R.O.C.

\section{NOMENCLATURE}

$\Delta E$ output emf of thermopile at a temperature difference $\Delta T, m v$

$E_{T}$ total output emf of thermopile, $m v$

$e(\Delta T)$ error in measurement of $\Delta T$ because of the uncertainty of estimating $T_{m},{ }^{\circ} \mathrm{C}$

$T$ temperature, ${ }^{\circ} \mathrm{C}$

$\Delta T \quad$ temperature difference $=T-T^{\prime},{ }^{\circ} \mathrm{C}$

$\epsilon$ output potential of thermocouple, $m v$

$\alpha_{A, i}$ absolute thermoelectric power of wire $\mathrm{A}, m v /{ }^{\circ} \mathrm{C}$

$\alpha_{A B, i}=\alpha_{A, i}-\alpha_{B, i}=$ Seebeck effect induced by the $i$ th pair of the thermopile, $m v /{ }^{\circ} \mathrm{C}$

\section{REFERENCES}

1. Huang, H., and Howell, J. R., Precision of Solar Collector Testing and Selection of Measurement Instruments, Solar Energy 35(5), 457-460, 1985. 
2. Schnurr, R. A., Thermopiles Aid in Measuring the Rate of Heat Rejection During Automotive Engine Tests, Genenal Motors Eng. J. 2, 8-11, 1963.

3. Baker, H. D., Ryder, E. A. and Baker, N. H., Temperature Measurement in Engineering, Vol. II, Chap. 3, Omega Press, Omega Engineering, Inc., Stamford, Conn., 1975.

4. Doebelin, E. O., Measurement Systems, Chap. 8, McGraw-Hill, New York, 1983.

5. Benedict, R. P., and Ashby, H. F., Empirical Determination of Ther- mocouple Characteristics, J. Eng. Power Trans. ASME, Jan., 9-26, 1963.

6. Kline, S. J., and McClintock, F. A., Describing Uncertainties in Single-Sample Experiments, Mech. Eng., 75, 3-8, 1953.

7. Moffat, R. J., Describing the Uncertainties in Experimental Results, Exp. Therm. Fluid Science, 1, 3-17 (1988).

Received September 26, 1988; revised May 3, 1989 